\title{
Coexpression of p53 and Ki 67 and lack of c-erbB2 expression in oral leukoplakias in India
}

Priya Kumar ${ }^{(a)}$

Shubhada Kane ${ }^{(\mathrm{b})}$

Govind Pomaji Rathod ${ }^{(\mathrm{c})}$

\footnotetext{
(a) Department of Oral and Maxillofacial Pathology, Maulana Azad Institute of Dental Sciences, New Delhi - 110002, Delhi, India.

(b) Department of Pathology, Tata Memorial Hospital, Lower Parel, Mumbai, India.

(c)Department of Oral and Maxillofacial Pathology, Rama Dental College, Kanpur, India.
}

Abstract: Oral cancer is commonly preceded by premalignant lesions and conditions. The clinician's ability to identify lesions at an increased risk of cancer development is critical for its control. The purpose of this study was to compare the expression of tumor suppressor gene p53, proliferation marker Ki-67, and oncogene c-erbB2 and to evaluate the relevance of their co-expression in the diagnosis of, and prognosis for, oral leukoplakia. In the present study, the expression of biomarkers was studied immunohistochemically in 55 cases of leukoplakia (26 without dysplasia, 29 with dysplasia) and 10 cases of normal epithelia. The Labeling Indices (LI) of p53 and Ki-67 were found to increase significantly with an increase in the grade of dysplasia. A significant correlation was also found between the LI of p53 and that of $\mathrm{Ki}-67$. It was also observed that c-erbB2 expression was only cytoplasmic, indicating incomplete receptor degradation. Hence, it can be concluded from the present study that the increased expression of p53 and Ki-67 with an increase in the grade of dysplasia suggests that their co-expression may be used for the identification of high-risk lesions. Also, c-erbB2 has no pathogenetic role in early carcinogenesis in the studied population, although incomplete receptor degradation, as evidenced by cytoplasmic staining, may indicate an early change.

Descriptors: Leukoplakia; Immunohistochemistry; Mouth Neoplasms; Oncogenes; Tumor Suppressor Protein p53.

\section{Introduction}

Oral cancer is most commonly associated with clinically definable premalignant lesions and conditions. In India, the most prevalent are leukoplakia and oral submucous fibrosis. ${ }^{1}$ The presence of epithelial dysplasia is generally accepted as one of the most important predictors of malignant development in premalignant lesions. However, the diagnosis of epithelial dysplasia is based on a static "snapshot". A better understanding of the fundamental molecular biology of the process of cancer development in the oral cavity through stages, conventionally defined as epithelial dysplasia/carcinoma in situ, may be the only way to improve our possibilities for predicting malignant development from precursor lesions. ${ }^{2}$

Activation of oncogenes, inactivation of tumor suppressor genes, and increased cellular proliferation are some of the key events in the multistep process of carcinogenesis. Located on chromosome 17p13.1 in the human genome, and encoding a $53-\mathrm{kDa}$ nuclear phosphoprotein, the p53 gene has been aptly

termed the "Guardian of the Genome". ${ }^{3}$ It is referred to as a tumor suppres-
Received for publication on Oct 07,2011 Accepted for publication on Mar 09, 2012
Corresponding author:

Priya Kumar

E-mail:drpri_kumar@yahoo.com 
sor gene because its normal function is to suppress the development of tumors by detecting genetic mistakes in G1 cells resulting in arrested cell growth (cell cycle arrest) or destruction (programmed cell death) of those cells. Alterations in the p53 tumor suppressor gene are among the most common genetic aberrations in the spectrum of oral cancers. ${ }^{4}$

$\mathrm{Ki}-67$ is a nuclear non-histone protein expressed maximally in cells in the $\mathrm{G} 2$ and $\mathrm{M}$ phases of the cell cycle, but is absent in resting cells. Hence, Ki-67 can be employed to measure the growth fraction of normal tissues, as well as premalignant and malignant lesions. ${ }^{5}$

The c-erbB2 proto-oncogene is a member of the Erb $\mathrm{B}$ family of transmembrane tyrosine kinase receptors. It can be activated by hetero-oligomerization with other members of the Erb family. This oncogene has been suggested to be significant with regard to inducing and maintaining oncogenesis in several human malignancies, and amplification of the c-erbB2 oncogene correlates with the immunohistochemical detection of the oncoprotein (p185) in primary carcinomas of the breast, ovary, stomach, pancreas, urinary bladder, kidney, oral cavity, and lungs, often with a significant association between c-erbB2 amplification and poor prognosis. ${ }^{6}$

This study was designed for the simultaneous evaluation and comparison of the expression of the tumor suppressor gene, p53, proliferation marker $\mathrm{Ki}-67$, and oncogene c-erbB2, in oral leukoplakias and their combined efficacy as predictors of high-risk lesions during screening procedures.

\section{Methodology}

The present study was done by a single investigator at the Government Dental College and Hospital, Mumbai (Protocol \#GDCHM 1-2006). The specimens were removed surgically from 55 patients (51 men and four women; ages ranging from 21 to 76 years, with a median of 47 years) having oral leukoplakia. Ten samples of normal oral epithelia were also evaluated as controls. Informed consent was obtained from all patients. The samples were subjected to routine histological processing followed by $\mathrm{H} \& \mathrm{E}$ staining. Based on the WHO criteria for histologic typing of cancer and precancer of the oral mucosa, they were then divided into lesions which exhibited keratosis or hyperplasia without dysplasia and dysplastic lesions with mild, moderate, and severe dys- plasia. Lesions having uniform color and surface characteristics were labeled "homogeneous", and those having mixed (red and white) and/or irregular surfaces were labeled as "non-homogeneous".

\section{Immunohistochemistry}

Sections of 5- $\mu \mathrm{m}$ thickness were prepared for each specimen, and immunohistochemical (IHC) analysis was carried out for each of the 3 primary antibodies. The sections were de-paraffinized and incubated in $0.6 \%$ methanol $\mathrm{H}_{2} \mathrm{O}_{2}$ for $15-20$ min to block endogenous peroxidase activity. Subsequently, slides were washed in $0.1 \mathrm{M}$ citric acid solution and irradiated in a microwave oven for $25 \mathrm{~min}$, allowed to cool for $20 \mathrm{~min}$, washed with Tris buffer, and prepared for immunohistochemical staining. Sections were covered with optimally diluted primary antibody anti-p53 (DO 7, Dako, Glostrup, Denmark), anti-Ki-67 (MiB-1, Dako), anti-c-erbB2 (CB 11, Novocastra, New Castle, UK), and negative reagent and incubated overnight at $4^{\circ} \mathrm{C}$, washed gently in buffer, and then incubated with biotinylated rabbit anti-mouse IgG (1:400 dilution) for $30 \mathrm{~min}$. Enough drops of streptavidin peroxidase (1:800 dilution) were applied to cover the specimen, which was then incubated for $45 \mathrm{~min}$, followed by the application of freshly prepared chromogen solution substrate and incubation at room temperature for 5-6 min. Sections were then counterstained with haematoxylin, dehydrated, and mounted.

\section{Quantitative analysis of IHC staining}

The expression of p53, Ki-67, and c-erbB2 was evaluated as follows:

- (for p53 and Ki-67) Brown nuclear staining was considered positive regardless of staining intensity. In the positive specimens, the Labeling Indices (LI) were calculated by manual counting of 500 cells in 5 different fields and the number of positive cells in each field. The percentage of positive cells was then calculated. The counting was done at $20 \times$ magnification.

- (for c-erbB2) Distinct membrane staining was considered as a positive reaction. The samples were graded according to the percentage of cells showing positive membrane stain. Cytoplasmic staining and granular membrane staining were considered negative. 
The following grading criteria were used:

- (1) None - No staining

- (2) $1+-<10 \%$ cells stained

- (3) $2+-10-50 \%$ cells stained

- (4) $3+->50 \%$ cells stained

\section{Statistical analysis}

All data were analyzed with SPSS (Statistical Package for the Social Sciences) software, version 16.0 (IBM, Chicago, USA). We applied the Kruskal-Wallis and Mann Whitney tests to assess the significance of differences within each group. We used Spearman's correlation coefficient to assess the correlation between the LI of p53 and that of Ki-67. The p-values of less than 0.05 were considered significant.

\section{Results}

Of the 55 cases examined, dysplasia was found in 29 (52.7\%). Mild dysplasia was observed in 19 (34.54\%), moderate in 7 (12.7\%), and severe in $3(5.4 \%)$ cases. The clinical types and sites of lesions did not correlate with the degree of dysplasia. The expression of both p53 and Ki-67 was observed as brown granular nuclear staining. The distribution of both biomarkers was limited to basal and parabasal layers in cases of leukoplakia without dysplasia, but extended to the suprabasal layers in cases with dysplasia. Distinct membranous c-erbB2 immunostaining was not seen in any of the cases. Although cytoplasmic staining was observed in many cases, it was considered negative. Positive controls were stained appropriately (Figures 1 through 4).

A statistically significant increase was observed in p53 LI ( $p=0.003)$ and Ki-67 LI ( $=0.002)$ with an increase in the grade of dysplasia. Also, based on Spearman's correlation coefficient, the mean p53 LI correlated significantly with the mean Ki-67 LI $(p=0.011)$ (Figure 5). However, when the LI were correlated with clinical types and sites, no statistically significant correlation could be found (Table 1).

\section{Discussion}

The clinician's ability to identify oral leukoplakia patients at increased risk of cancer development is critical for improving control of oral cancer. Although dysplastic changes are often found adjacent to foci of cancerous transformation, and long-term studies have shown that epithelial dysplasia almost invariably antedates the appearance of cancer, the term "dysplasia" without qualification does not indicate cancerous transformation, and dysplasias do not necessarily progress to cancer. ${ }^{6}$ Also, diagnosis of epithelial dysplasia is subjective, and thus
Table 1 - Relationship between clinicopathologic features and labeling indices of $\mathrm{p} 53$ protein and Ki-67 antigen.

\begin{tabular}{|c|c|c|c|c|c|}
\hline & Number & $\mathrm{p} 53$ & Significance & Ki 67 & Significance \\
\hline \multicolumn{6}{|c|}{ Dysplasia* } \\
\hline Normal epithelium & 10 & $1.88 \pm 1.69$ & \multirow{5}{*}{$\mathrm{P}<0.0001$} & $6.38 \pm 2.32$ & \multirow{5}{*}{$\mathrm{P}<0.0001$} \\
\hline No dysplasia & 26 & $4.47 \pm 5.00$ & & $9.802 \pm 6.3691$ & \\
\hline Mild dysplasia & 19 & $8.75 \pm 6.40$ & & $13.559 \pm 6.3171$ & \\
\hline Moderate dysplasia & 7 & $9.88 \pm 7.38$ & & $17.836 \pm 1.9871$ & \\
\hline Severe dysplasia & 3 & $24.54 \pm 16.60$ & & $21.100 \pm 7.0612$ & \\
\hline \multicolumn{6}{|c|}{ Site* } \\
\hline Buccal mucosa & 45 & $7.004 \pm 8.1387$ & \multirow{5}{*}{0.093} & $11.5 \pm 6.5879$ & \multirow{5}{*}{0.086} \\
\hline Palate & 2 & $9.415 \pm 8.9025$ & & $18.71 \pm 4.3982$ & \\
\hline Labial mucosa & 4 & $14.775 \pm 5.4920$ & & $18.17 \pm 7.1969$ & \\
\hline Tongue & 3 & $10.763 \pm 2.8789$ & & $18.597 \pm 3.0554$ & \\
\hline Alveolar mucosa & 1 & 0 & & $17.230 \pm 0$ & \\
\hline \multicolumn{6}{|c|}{ Clinical Type\# } \\
\hline Homogeneous & 37 & 26.43 & \multirow{2}{*}{0.293} & 27.09 & \multirow{2}{*}{0.548} \\
\hline Non-homogeneous & 18 & 31.22 & & 29.86 & \\
\hline
\end{tabular}

*Kruskal-Wallis test; \#Mann-Whitney U-test; $\mathrm{p}<0.05$ 


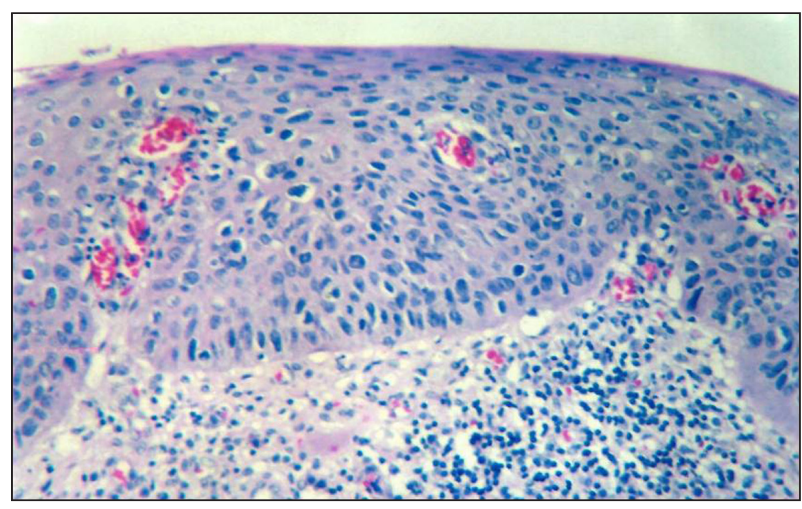

Figure 1 - Leukoplakia showing moderate epithelial dysplasia. Original magnification $200 \times$.

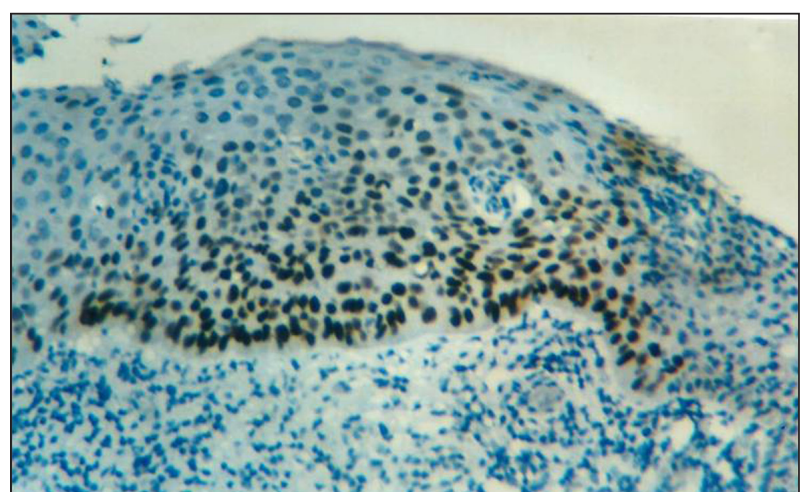

Figure 2 - Strong p53 positivity with distribution of positive cells from basal layer to superficial epithelial layers in a case with moderate epithelial dysplasia. Original magnification $200 \times$.

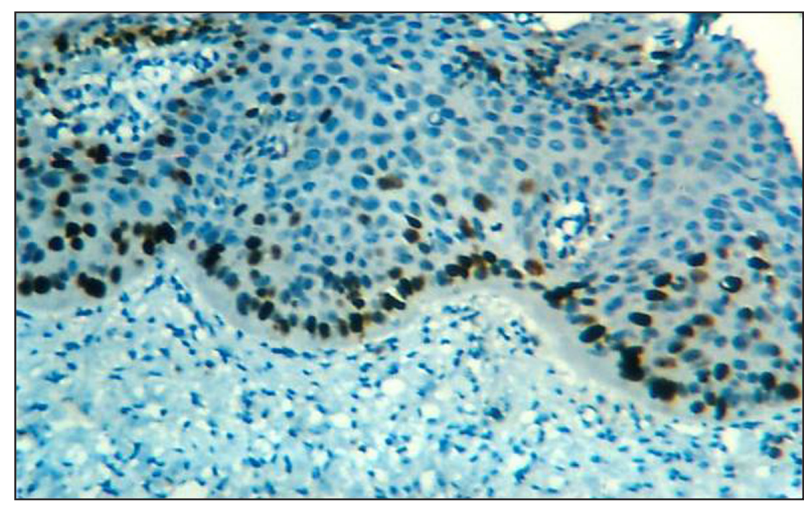

Figure 3 - Strong Ki67 positivity seen in the basal layer with few positive cells in the superficial layers. Original magnification $200 \times$.

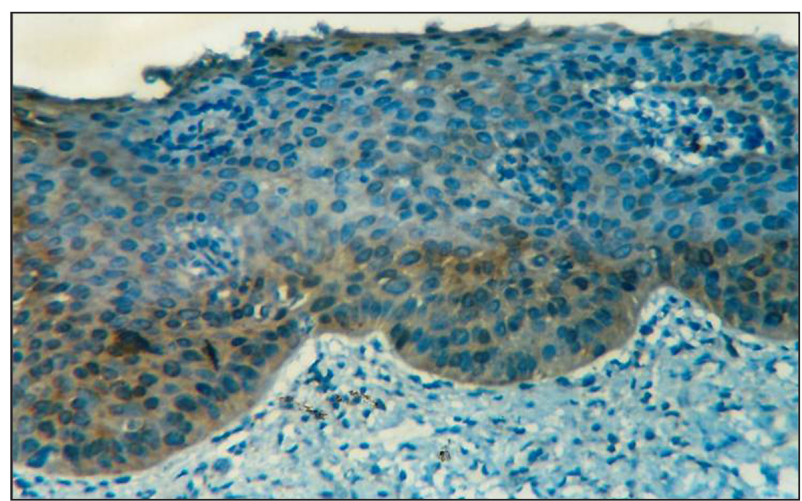

Figure 4 - Negative expression of c-erbB2 in the same case. Diffuse cytoplasmic staining is observed. Original magnification $200 \times$.

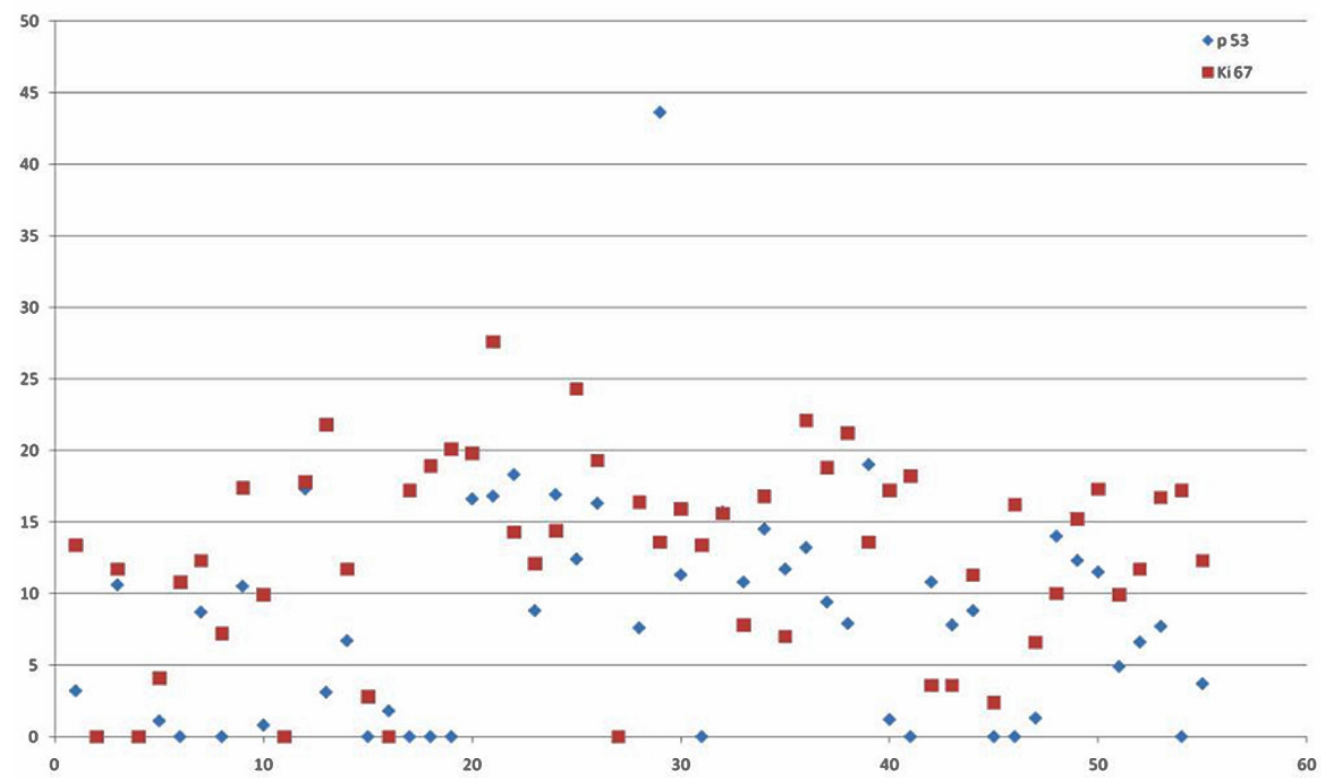

Figure 5 - Spearman's correlation coefficient showing positive correlation between $\mathrm{p} 53$ and $\mathrm{Ki} 67$. 
the inclusion of molecular biology techniques in the detection of premalignant lesions may ostensibly improve the detection of alterations that are invisible to the microscope.? Out of the vast array of molecular markers available, markers for tumor growth and those for tumor suppression have been found to have the most prognostic relevance. ${ }^{8}$ We studied the expression of c-erbB2 and Ki-67 (markers for tumor growth), the expression of p53 (a marker of tumor suppression under normal conditions), and the relevance of their co-expression in the diagnosis of, and prognosis for, oral leukoplakia.

Under normal circumstances, p53 protein prevents the propagation of genetically damaged cells by assisting in DNA repair, and alterations in this gene have been found to be the most common event in most human malignancies, including oral cancer. ${ }^{9}$ In this study, p53 was found to be positive in 40 of the 55 (72.7\%) cases of leukoplakia. Similar observations have been made in many other studies. ${ }^{10-12}$ The finding of p53 mutations associated with an invasive phenotype in oral squamous cell carcinoma suggests the possibility that this change can be used for the molecular screening of oral premalignant lesions. This approach may provide an opportunity for the better monitoring of patients in whom the development of oral cancer is unpredictable and the majority of whom, despite similar histology, will not develop progressive disease. ${ }^{13}$ The immunohistochemical expression of p53 in smokeless tobacco users, for example, has been found to be discordant in various studies.

Since the present study involved subjects indulging in various forms of tobacco abuse-i.e., smokeless, smoking, and a combination of both forms - the study of the mutational status of 553 may be of more prognostic significance. This especially holds true for p53 immunostaining, wherein it is difficult to determine whether positive staining is a consequence of p53 mutation or an elevated "wild" p53 expression, where p53 protein is bound to another protein and retained in the tissue but in an inactive state. ${ }^{14}$ At the same time, it is important to emphasize that immunohistochemical identification of p53 almost certainly implies a protein with a defective function because of either mutation or inactivation. ${ }^{15}$

Along with the activity of tumor suppressor genes, tumor differentiation and proliferative activity are also important predictors of biologic behavior, and cell proliferation markers may be helpful in assessing the types and degrees of oral premalignant and malignant lesions. The "S-phase fraction" correlates with the growth fraction and is indicative of the proliferative capacity of the cell, which can be measured by immunohistochemical staining with Ki-67. ${ }^{16}$ In our study, we observed Ki-67 positivity in 49 of the 55 cases.

Amplification of the c-erbB2 gene has been seen in many human malignancies, but the relevance of its expression in head and neck squamous cell carcinoma is controversial. ${ }^{17,18}$ While c-erbB2 membrane expression has been correlated with gene amplification, the significance of cytoplasmic c-erbB2 expression, which was largely observed in our cases, is not exactly known but has been postulated to represent incomplete receptor degradation. ${ }^{19}$ In the literature, the results of c-erbB2 immunostaining have been reported to be extremely variable, since both cytoplasmic staining and membranous staining are considered positive. ${ }^{18}$

In this study, distinct c-erbB2 membrane staining was not observed in any case. Although cytoplasmic staining was noted in many cases, it was not considered positive, since the manufacturer's instructions for interpretation recommended that cases with only cytoplasmic staining should be considered negative. ${ }^{16}$ Our findings are similar to those of Liu et al. ${ }^{20}$

However, $\mathrm{Hou}^{21}$ et al. and Wilkman et al. ${ }^{22}$ reported a progressive increase in c-erbB2 expression with an increase in the grade of epithelial dysplasia. This positive staining reaction could be due to differences in their criteria, i.e., their inclusion of cytoplasmic staining as a positive reaction.

The staining patterns of $\mathrm{p} 53$ and Ki67 were found to be similar. A statistically significant increase in the LI of p53 and Ki67 was noted with increasing grades of dysplasia in oral leukoplakias. Also, the staining was restricted to the basal cell layer in non-dysplastic leukoplakias, with an increase in suprabasal positivity as the grade of dysplasia increased. Such a pattern of staining was also observed by Cruz et al., ${ }^{23,24}$ Kerdpon et al., ${ }^{11}$ Vered et al. ${ }^{15}$ and Nasser et al. ${ }^{25}$ These investigators also found that the p53 expression pattern was significantly related to the development of carcinoma. Many previous studies have reported similar observations. ${ }^{26-28}$ Nasser et al. ${ }^{25}$ found $\mathrm{p} 53$ and $\mathrm{Ki}-67$ to have low positive predictive value when expressed independently, but their dual expression could help discriminate between dysplastic and 
non-dysplastic leukoplakias. Gouvea et al. ${ }^{29}$ found p53 and $\mathrm{Ki}-67$ expression in only a few cases of mild and moderately dysplastic proliferative verrucous leukoplakias (PVL), and their immunoexpression did not correlate with progressive epithelial alterations. This discrepancy may be explained by the fact that PVL is a distinct lesion predominating in females and having an etiology other than the use of tobacco.

Under normal circumstances, p53 regulates the cell cycle by inducing G1 arrest or apoptosis in cells that are genetically damaged by ultraviolet light or chemical carcinogens. ${ }^{30}$ Mutations of p53 lead to uncontrolled cell growth. Thus, the significant correlation between p 53 and $\mathrm{Ki}-67$ in our study indicated that alterations in p53 may lead to increased cellular proliferation in premalignant lesions. Also, cytoplasmic and granular membranous expression of c-erbB2 may be an early indicator of oncogene activation in premalignancy.

\section{Conclusion}

The expression of $\mathrm{p} 53$ and Ki-67 increased with increasing grades of dysplasia, indicating that they may be used as predictive markers in oral cancer development. Also, the expression of p53 correlated significantly with Ki-67, suggesting that altered $\mathrm{p} 53$ gene product promoted cellular proliferation. Lack of c-erbB2 expression in oral leukoplakia suggested that it does not play a pathogenetic

9. Levine AJ. p53, the cellular gate keeper for growth and division. Cell. 1997 Feb;88(3): 323-31.

10. Agarwal S, Mathur M, Srivastava A, Ranju R. MDM2/p53 coexpression in oral premalignant and malignant lesions: potential prognostic implications. Oral Oncol. 1999 Mar;35(2):209-16.

11. Kerdpon D, Rich AM, Reade PC. Expression of p53 in oral mucosal hyperplasia, dysplasia and squamous cell carcinoma. Oral Dis. 1997 Mar;3(2):86-92.

12. Murti PR, Warnakulasuriya KAAS, Johnson NW, Bhonsle RB, Gupta PC, Daftary DK, et al. p53 expression in oral precancer: a marker for malignant potential. J Oral Pathol Med. 1998 May;27(5):191-6.

13. van Oijen MGCT, van de Craats JG, Slootweg PJ. p53 overexpression in oral mucosa in relation to smoking. J Pathol. 1999 Mar;187(4):469-74.

14. Warnakulasuriya KAAS, Ralhan R. Clinical, pathological and molecular lesions caused by oral smokeless tobacco - a review. J Oral Pathol Med. 2007 Feb;36(2):63-77. role in the early stages of carcinogenesis in this Indian population. However, incomplete receptor degradation, as evidenced by cytoplasmic staining, may indicate an early change in oral cancer development.

\section{References}

1. Mehta FS, Gupta PC, Daftary DK, Pindborg JJ, Choksi SK. An epidemiologic study of oral cancer and precancerous conditions among 101,761 villagers in Maharashtra, India. Int J Cancer. 1972 Jul;10(1):134-41.

2. Reibel J. Prognosis of oral premalignant lesions: Significance of clinical, histopathological, and molecular biological characteristics. Crit Rev Oral Biol Med. 2003 Jan;14(1):47-62.

3. Lane DP. p53, guardian of the genome. Nature. 1992 Jul;358(6381):156.

4. Sarnath D, Tandle AT, Teni TR, Dedhia PM, Borges AM, Parikh $\mathrm{D}$, et al. $\mathrm{p} 53$ inactivation in chewing tobacco-induced oral cancers and leukoplakias from India. Oral Oncol. 1999 May;35(3):242-50.

5. Iamaroon A, Khemaleelakul U, Pongsiriwet S, Pintong J. Coexpression of p53 and Ki 67 and lack of EBV expression in oral squamous cell carcinoma. J Oral Pathol Med. 2004 Jan;33(1):30-6.

6. Hynes NE. Amplification and overexpression of the erbB-2 gene in human tumors: its involvement in tumor development, significance as a prognostic factor, and potential as a target for cancer therapy. Semin Cancer Biol. 1993 Feb;4(1):19-26.

7. Chimenos-Kustner E, Font-Costa I, Lopez-Lopez J. Oral cancer risk and molecular markers. Med Oral Patol Oral Cir Bucal. 2004 Nov-Dec;9(5):377-84.

8. Schliephake H. Prognostic relevance of molecular markers of oral cancer - a review. Int J Oral Maxillofac Surg. 2003 Jun;32(3):23345 .

15. Vered M, Allon I, Dayan D. Maspin, p53, and Ki- 67 in epithelial lesions of the tongue: from hyperplasia to dysplasia to carcinoma. J Oral Pathol Med. 2009 Mar;38(3):314-20.

16. Girod SC, Pfeiffer P, Ries J, Pape HD. Proliferative activity and loss of function of tumour suppressor genes as biomarkers in diagnosis and prognosis of benign and preneoplastic oral lesions and oral squamous cell carcinoma. Br J Oral Maxillofac Surg. 1998 Aug;36(4):252-60.

17. Yamamoto T, Ikawa S, Akiyama T, Semba K, Nomura N, Miyajima $\mathrm{N}$, et al. Similarity of protein encoded by the human c-erb-B-2 gene to epidermal growth factor receptor. Nature. 1986 Jan;319(6050):230-4.

18. Del Sordo R, Angiero F, Bellezza G, Cavaliere A, Mameli M-G, Stefani M, et al. HER family receptors expression in squamous cell carcinoma of the tongue: study of the possible prognostic and biologic significance. J Oral Pathol Med. 2010 Jan;39(1):79-86. 
19. Ibrahim SO, Lillehaug JR, Johannessen AC, Liavaag PG, Nilsen R, Vasstrand EN, et al. Expression of biomarkers (p53, transforming growth factor alpha, epidermal growth factor receptor, c-erbB-2/ neu and the proliferative cell nuclear antigen) in oropharyngeal squamous cell carcinoma. Oral Oncol. 1999 May;35(3):302-13.

20. Liu X, Han Z, Wang Y. Xing jiao yuan he c-erbb-2 zai kou qiang nian zhu jun bai ban zhong de biao da gao yan. Zhonghua Kou Qiang Yi Xue Za Zhi. 1999 Nov.;34(6):325-7.

21. Hou L, Shi D, Tu SM, Zhang HZ, Hung MC, Ling D. Oral cancer progression and c-erb-B2/neu proto-oncogene expression. Cancer Lett. 1992 Aug;65(3):215-20. Complement.

22. Wilkman TSE, Hietanen JPH, Malmstrom MJ, Konttinen YT. Immunohistochemical analysis of the oncoprotein c-erb-B2 expression in oral benign and malignant lesions. Int J Oral Maxillofac Surg. 1998 Jun;27(3):209-12.

23. Cruz I, Napier SS, van der Waal I, Snijders PJF, Walboomers JMM, Lamey PJ, et al. Suprabasal p53 immunoexpression is strongly associated with high grade dysplasia and risk for malignant transformation in potentially malignant oral lesions from Northern Ireland. J Clin Pathol. 2002 Feb;55(2):98-104.

24. Cruz IB, Snijders PF, Meijer CJ, Braakhuis BJ, Snow BG, Walbloomers JM, et al. p53 expression above the basal cell layer in oral mucosa is an early event of malignant transformation and has predictive value for developing oral squamous cell carcinoma. J Pathol. 1998 Apr;184(4):360-8.

25. Nasser W, Flechtenmacher C, Holzinger D, Hofele C, Bosch FX. Aberrant expression of $\mathrm{p} 53$, p16 $6^{\mathrm{INK} 4 \mathrm{a}}$ and $\mathrm{Ki}-67$ as basic biomarker for malignant progression of oral leukoplakias. J Oral Pathol Med. 2011 Sep;40(8):629-35.

26. Kurokawa H, Matsumoto S, Murata T, Yamashita Y, Tomoyose T, Zhang M et al. Immunohistochemical study of syndecan-1 downregulation and expression of 553 protein or $\mathrm{Ki}-67$ antigen in oral leukoplakia with or without epithelial dysplasia. J Oral Pathol Med. 2003 Oct;32(9):513-21.

27. Girod SC, Krueger G, Pape HD. p53 and Ki-67 expression in preneoplastic and neoplastic lesions of the oral mucosa. Int J Oral Maxillofac Surg. 1993 Oct;22(5):285-8.
28. Takeda T, Sugihara K, Hirayama Y, Hirano M, Tanuma JT. Immunohistological evaluation of Ki67, p63, CK 19 and p53 expression in oral epithelial dysplasias. J Oral Pathol Med. 2006 Jul;35(6):369-75.

29. Gouvea AF, Vargas PA, Coletta RD, Jorge J, Lopes MA. Clinicopathological features and immunohistochemical expression of $\mathrm{p} 53$, $\mathrm{Ki}-67, \mathrm{Mcm}-2$ and Mcm-5 in proliferative verrucous leukoplakia. J Oral Pathol Med. 2010 Jul;39(6):447-52.

30. Chang F, Syrjanen S, Syrjanen K. Implications of the p53 tumor suppressor gene in clinical oncology. J Clin Oncol. 1995 Apr;13(4):1009-22. 